\title{
Eficiencia en la remoción de materia orgánica mediante lombrifiltros (Eisenia foetida) en aguas residuales domésticas para zonas rurales
}

\section{(Efficiency in the removal of organic matter by wormfilters (Eisenia foetida) in domestic wastewater for rural areas)}

\author{
Jonathan Gustavo Castillo Sánchez¹, Jessica Estefanía Chimbo Solórzano²
}

\begin{abstract}
Resumen
El inadecuado manejo de las aguas residuales domésticas en zonas rurales vertidas en cuerpos hídricos sin previo tratamiento constituye un gran problema de polución ambiental, así como un riesgo para la salud pública. El propósito del este estudio fue evaluar la eficiencia de la remoción de materia orgánica mediante lombrifiltros en las aguas residuales domésticas de una zona rural. En condiciones experimentales, se diseñó el lombrifiltro utilizando como base teórica el sistema de tratamiento Tohá - creado por el Dr. José Tohá-conformado por cuatro estratos: aserrín + Eisenia foetida, carbón activado, grava y piedras de río. Se evaluó la concentración de materia orgánica del afluente obteniendo elevadas concentraciones de $\mathrm{DBO}_{5}$, DQO, SST y ST. T 1 alcanzó los mayores porcentajes de eficiencia con un flujo volumétrico de $1.8 \times 10^{-2} \mathrm{l} / \mathrm{s}$ y TRH de 0.92 h obteniendo $52.25 \%$ para DBO y DQ0, $66.74 \%$ de SST y $52.91 \%$ de ST. De esta manera, se determinó que la utilización de lombrifiltros a base de Eisenia foetida para remover la materia orgánica representa un sistema de tratamiento ecológico e innovador que requiere una baja inversión, es eficiente y amigable con el ambiente.
\end{abstract}

\section{Palabras clave}

Lombrifiltro, Eisenia foetida, aguas residuales domésticas, caudal, eficiencia.

\begin{abstract}
Inadequate management of domestic wastewater in rural areas, the discharge of which to water bodies without prior treatment, is a major problem of environmental pollution, as well as a risk to public health. The purpose of this study was to assess the efficiency in the removal of organic matter by lombrifilters in domestic wastewater in a rural area. Under experimental conditions, the wormfilter was designed using as a theoretical basis the Tohá treatment system, created by Dr. José Tohá, which consisted of four strata: sawdaught + Eisenia foetida, activated charcoal, gravel and river stones. The concentration of organic matter of the tributary was evaluated obtaining high concentrations of $B O D_{5^{\prime}}$ COD, TSS and TS. T1 achieved the highest efficiency percentages with a volumetric flow of $1.8 \times 10^{-2} \mathrm{l} / \mathrm{S}$ and TRH of $0.92 \mathrm{~h}$ obtaining $52.25 \%$ for $\mathrm{BOD}_{5}$ and $\mathrm{COD}, 66.74 \%$ TSS and $52.91 \%$ TS. In this way, it was determined that the implementation of wormfilters based on Eisenia foetida for the removal of organic matter, represents an ecological and innovative treatment system that requires a low investment, is efficient and friendly to the environment.
\end{abstract}

\section{Keywords}

Worm filter, Eisenia foetida, domestic wastewater, flow, efficiency.

\footnotetext{
1 Escuela Superior Politécnica Agropecuaria de Manabí Manuel Félix López. Calceta, Ecuador [jonathan.castillo@espam.edu. ec, https://orcid.org/0000-0002-9932-7868].

2 Escuela Superior Politécnica Agropecuaria de Manabí Manuel Félix López. Calceta, Ecuador [jessica.chimbo@espam.edu.ec, https://orcid.org/0000-0001-9424-3430].
} 


\section{Introducción}

Actualmente, con la rápida urbanización y el crecimiento acelerado de la población, uno de los principales problemas de contaminación es el inadecuado manejo de las aguas residuales domésticas cuya descarga a cuerpos de agua y su reutilización sin un tratamiento previo representa un problema de contaminación de los recursos hídricos, pérdida de ecosistemas y un potencial riesgo para la salud pública (Cáceres et al., 2018; Yang et al., 2020). El 90 \% de las aguas residuales se vierten a los sistemas de agua sin tratamiento en los países en desarrollo. Los estudios demuestran que cada año se vierten al agua unas 730 megatoneladas de residuos procedentes de las aguas residuales y otros vertidos (Priya et al., 2021). En 2016, el tratamiento de las aguas residuales domésticas en las aldeas rurales de China era menos del $17.4 \%$ (NBS, 2017). La gran inversión requerida para instalar un sistema de tratamiento de aguas residuales convencional, el alto consumo de energía y los costos de mantenimiento y operación son las principales razones por las que esta alternativa no tiene éxito en comunidades rurales de países latinoamericanos, como en el caso de Ecuador (De Anda, 2017). En efecto, un elevado número de familias descargan sus aguas residuales directamente en el suelo o en los cuerpos de agua sin previo tratamiento, ocasionando así una polución ambiental y consecuentemente afectaciones en la salud (Dharwal et al., 2020; Reyes, 2016).

Las diferencias marcadas en los aspectos socioeconómicos, ambientales y tecnológicos reducen considerablemente el acceso a sistemas o instalaciones mínimas necesarias para depurar las aguas residuales, específicamente, en las zonas rurales (Lohani et al., 2020). Por tal motivo, hay una necesidad urgente de emplear alternativas innovadoras, eficientes y económicas para tratar estas aguas (Sosa et al., 2014; Tejada et al., 2018). La vermifiltración y especialmente el Sistema Tohá, a partir de lombrifiltros, son los procesos de tratamiento de aguas residuales mediante una acción conjunta de lombrices de tierra y microorganismos, minimizando considerablemente la carga orgánica del agua, así como otros contaminantes físicos, químicos y biológicos (Ramón et al., 2015).

Es decir, es una extensión del proceso de vermicompostaje; es un biofiltro donde las lombrices de tierra digieren las partículas en suspensión tamizadas en el lecho filtrante y degradan la materia orgánica mediante actividad enzimática y en proceso de ingestión; airean pasivamente el sistema mediante la acción de excavación y eliminan los patógenos (Sudipti \& Sakshi, 2021). De tal manera, podría ser una tecnología ideal para tratar los efluentes industriales y domésticos, por sus características rentables y ecológicamente sostenibles (Singh et al., 2017).

Debido a que existe una escasez de métodos naturales utilizados como alternativas para tratar las aguas residuales domésticas en zonas rurales de Manabí, este estudio busca diseñar e implementar un sistema de tratamiento ecológico e innovador basado en lombrifiltros con Eisenia foetida que requieran una baja inversión, que sean eficiente y amigables con el ambiente.

\section{Materiales y métodos}

\section{1. Área de estudio}

Tal como se muestra en la Figura 1, el estudio se llevó a cabo en CIIDEA (Ciudad de Investigación, Innovación y Desarrollo Agropecuario) de la ESPAM MFL (Escuela Superior Politécnica Agropecuaria de Manabí Manuel Félix López), situada en el sitio El Limón, parroquia Calceta (Bolívar, 
Manabí). Los análisis de laboratorio se hicieron en el laboratorio de Química Ambiental del Área Agroindustrial de la ESPAM MFL y en el de Investigación de la Universidad de las Américas (Quito). Las muestras de agua fueron tomadas de la comunidad El Limón Adentro, parroquia Calceta, cantón Bolívar, provincia de Manabí.

Figura 1. Mapa de ubicación

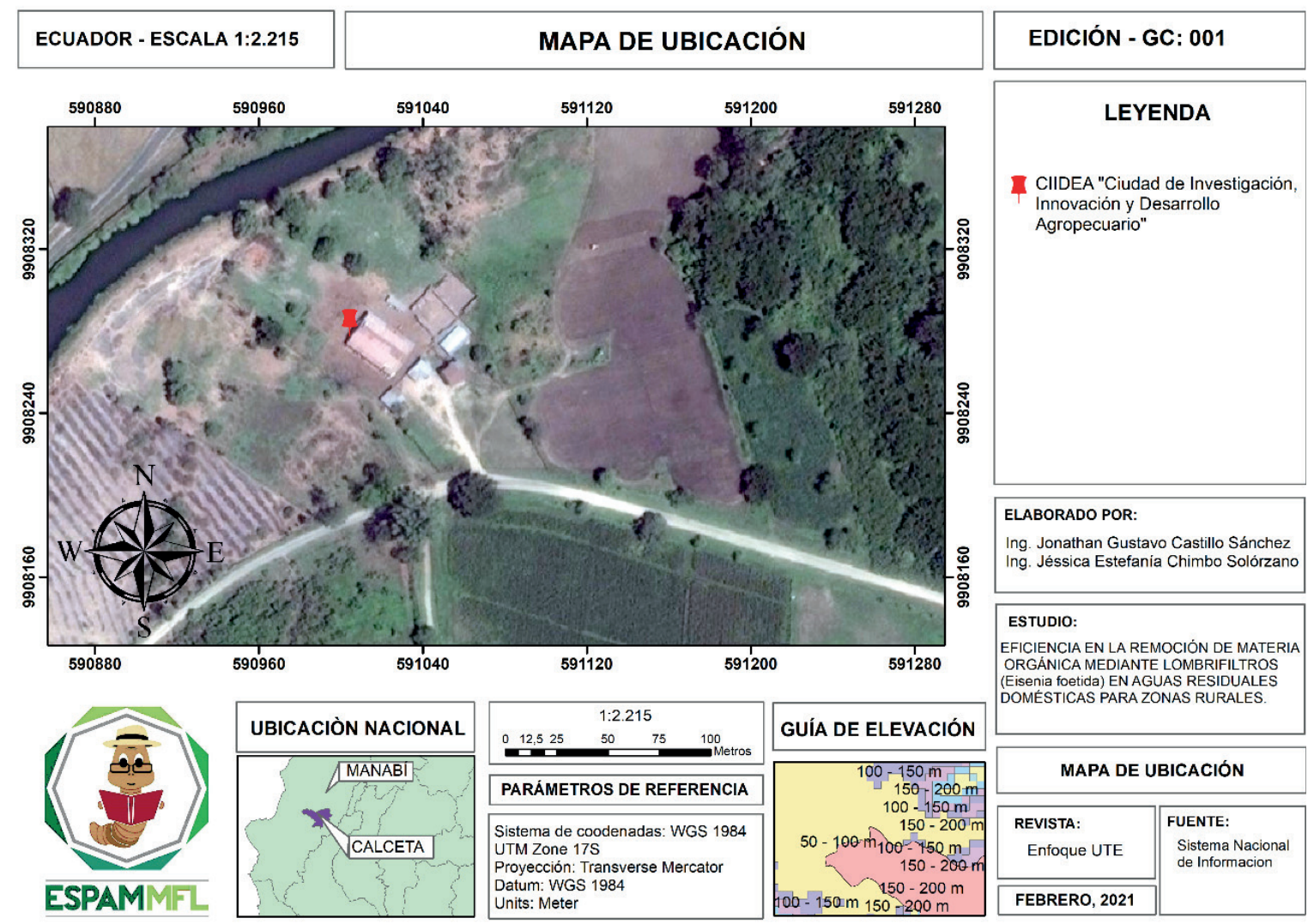

\subsection{Diseño experimental}

Se elaboró un diseño experimental completamente al azar con un solo factor (agua residual doméstica), contando con tres tratamientos y con tres repeticiones, donde se obtuvo un total de nueve unidades experimentales, las cuales son las muestras del agua residual doméstica tratada (Tabla 1). Estos tratamientos están constituidos por el caudal (l/s) que es manipulado con diferentes aperturas de la válvula de control, la que pudiera influir directamente en el tiempo de retención hidráulico medido en horas. Asimismo, se hicieron pruebas de Tukey y gráficos estadísticos mediante el software InfoStat y Microsoft Excel. 
Tabla 1. Diseño experimental

\begin{tabular}{|c|c|c|}
\hline Tratamiento & Unidades experimentales & Apertura de la válvula de control \\
\hline \multirow{3}{*}{$\mathrm{T}_{1}$} & $\mathrm{R}_{1}$ & \multirow{3}{*}{$25 \%$} \\
\hline & $\mathrm{R}_{2}$ & \\
\hline & $\mathrm{R}_{3}$ & \\
\hline \multirow{3}{*}{$\mathrm{T}_{2}$} & $\mathrm{R}_{1}$ & \multirow{3}{*}{$50 \%$} \\
\hline & $\mathrm{R}_{2}$ & \\
\hline & $\mathrm{R}_{3}$ & \\
\hline \multirow{3}{*}{$\mathrm{T}_{3}$} & $\mathrm{R}_{1}$ & \multirow{3}{*}{$100 \%$} \\
\hline & $\mathrm{R}_{2}$ & \\
\hline & $\mathrm{R}_{3}$ & \\
\hline
\end{tabular}

\subsection{Toma de muestras del agua residual doméstica}

La toma de muestras se llevó a cabo en una vivienda de la comunidad El Limón Adentro, parroquia Calceta, cantón Bolívar, provincia de Manabí. Esta se hizo a las 14h00, ya que García (2012) recomienda tomar las muestras en las horas donde existe mayor actividad y movimiento de las personas, para que la concentración de la materia orgánica del agua residual sea la adecuada para el tratamiento.

El procedimiento utilizado para el muestreo se hizo con base en la Norma Técnica Ecuatoriana NTE INEN 2176:2013 (Agua. Calidad del agua, 2013b), para lo cual fue necesario purgar los envases tres veces antes de recolectar la muestra. Se transportaron las muestras en un cooler y fueron almacenadas, en refrigeración, a $4^{\circ} \mathrm{C}$, de las cuales 5 I fueron utilizados para el análisis en laboratorio, 10 I para el proceso de adaptación del material biológico (Eisenia foetida) y 60 I fueron utilizados para el tratamiento en el lombrifiltro.

El tipo de recipiente que se utilizó para la toma de muestras fue seleccionado de acuerdo con la Norma Técnica Ecuatoriana NTE INEN 2169:2013 (Agua. Calidad del agua, 2013a), establecidos en la Tabla 1: Técnicas generales para la conservación de muestras - análisis físico-químico.

\subsection{Diseño y construcción del lombrifiltro}

El lombrifiltro fue diseñado con base en el estudio realizado por Ramón et al. (2015) quienes indican que se tiene un tanque alimentador de $60 \mathrm{l}$, el cual vierte en el lombrifiltro el agua residual. El agua pasa por un tubo de PVC agujerado para crear un caudal continuo y estable. De esta manera, el agua residual pudo ser repartida homogéneamente al sustrato del lombrifiltro. Considerando el estudio de Samal et al. (2018), en el diseño se tuvieron en cuenta los siguientes parámetros: caudal, tiempo de retención hidráulico, profundidad, ancho y largo de la caja, y altura del sistema.

Los lechos fueron clasificados de la siguiente forma: aserrín con lombriz, seguido de un lecho con carbón activado, pasando luego por un lecho de grava, para finalmente colocar un le- 
cho de piedra de río; permitiendo filtrar y oxigenar el líquido sin que genere malos olores. Debido a que el proceso es aerobio, el agua filtrada por cada uno de los lechos filtrantes se recolectó en el último recipiente que fue usado para la toma de muestras de laboratorio.

La altura de las capas del lombrifiltro se calculó con la ecuación propuesta por Coronel (2015):

$$
h=\frac{\% \text { total del estrato } * \text { altitud útil del lombrifiltro }}{100}
$$

En la Figura 2 se muestran las dimensiones del lombrifiltro, donde las medidas internas respectivamente son las siguientes: ancho $25 \mathrm{~cm}$, largo $44 \mathrm{~cm}$ y altura $25 \mathrm{~cm}$. Para ello se tomó en cuenta el estudio de Salazar (2005), en el que se recomienda que la parte biológica debe contener el mayor porcentaje; por lo tanto, se dispuso de un $40 \%$ para la primera capa de material biológico (Eisenia foetida+aserrín), seguido de un 20 \% para los estratos de carbón activado, grava y piedra.

Figura 2. Diseño del lombrifiltro

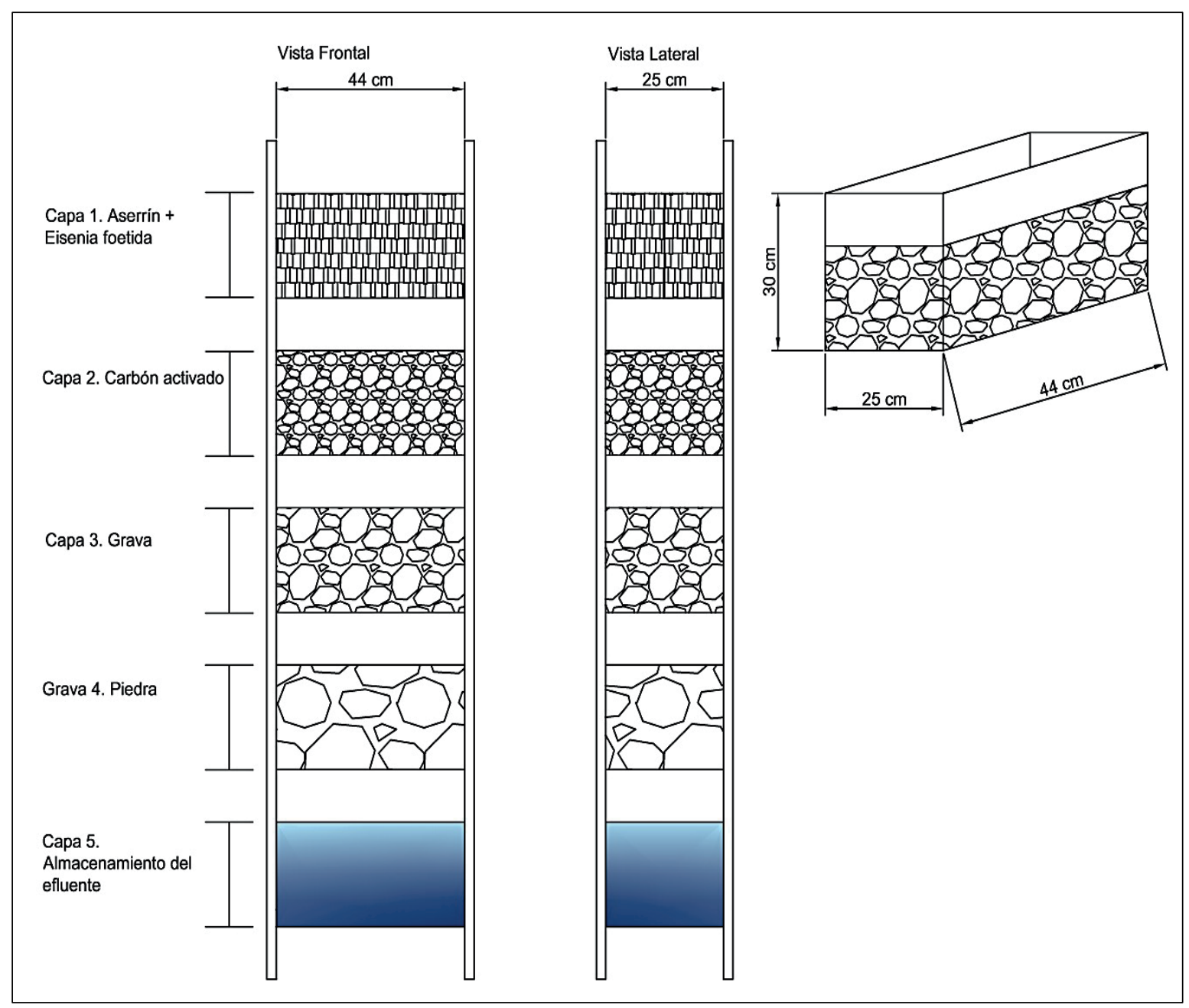




\subsection{Adaptación de Eisenia foetida al agua residual doméstica}

Las lombrices fueron recolectadas de las camas de lombricultura de CIIDEA-ESPAM MFL. Se hizo una excavación de aproximadamente $30 \mathrm{~cm}$ de profundidad, de donde se las recolectó, mediante una lampa, y, luego, se las separó de la tierra que se encontraba impregnada en las ellas. Se utilizó 1 kg de lombrices para el proceso de remoción.

Antes de colocar a las lombrices Eisenia foetida en el lombrifiltro para iniciar el proceso tratamiento de las aguas residuales, fue necesario que pasen por un proceso de aclimatación para que lograran adaptarse, de manera progresiva, a estar en contacto con las aguas residuales; de lo contrario, exponerlas de manera directa dañaría la epidermis de las lombrices, incluso podrían experimentar cambios bruscos de su entorno o hábitat que afecten su metabolismo (Figura 3).

Con base en la investigación de Loro (2018), el proceso de adaptación fue de 10 días. Este consistió en regar diariamente 1 I de agua residual doméstica a una caja con sustrato (humus) donde habitaban todas las lombrices; dicha caja tenía agujeros en la base y una malla plástica tipo Raschell, para evacuar el exceso de agua por infiltración, pero, a su vez, mantenía húmedo el sustrato necesario para la respiración de las lombrices.

Guzmán et al. (2020) recomiendan que en este periodo de aclimatación se debe evaluar la reproducción y el acondicionamiento de las lombrices Eisenia foetida en el sustrato orgánico que se utilizará, controlando su pH, porcentaje de humedad y temperatura.

Figura 3. Adaptación de la Eisenia foetida

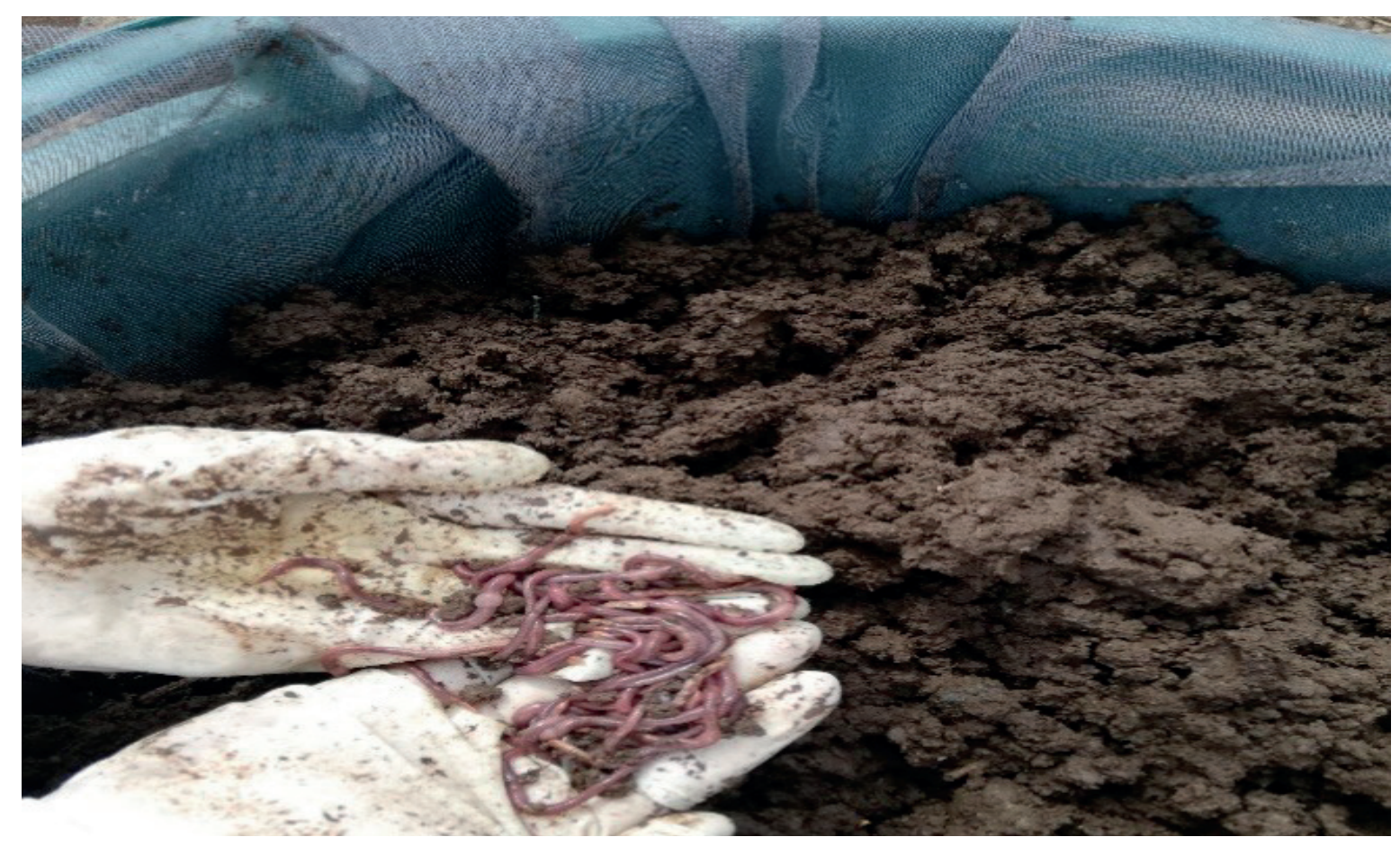

\subsection{Medición del caudal y tiempo de retención hidráulico (TRH)}

Para la medición del caudal se empleó el método volumétrico. Se abrió la llave de control del tanque alimentador y se colocó un recipiente de 1 I debajo de las tuberías que asperjan el agua, 
se recolectó el agua residual durante 1 minuto y se retiró el recipiente con el agua recolectada. Posteriormente, se midió la cantidad de agua residual recolectada; esta acción se repitió tres veces para obtener resultados más acertados. Los tiempos de retención hidráulico (TRH) fueron medidos como el tiempo que demora el agua en pasar por el estrato e iniciar su aparición en el fondo de este, empleando cada uno de los caudales seleccionados. Para ello se utilizó la ecuación propuesta por Coronel (2015):

$$
\boldsymbol{T R H}=\frac{V}{Q}
$$

\subsection{Determinación de la eficiencia (porcentaje de remoción)}

Para evaluar el funcionamiento del prototipo biológico compuesto de Eisenia foetida, se determinó el porcentaje de eficiencia de los parámetros analizados con la ecuación propuesta por Caicedo (2017), la cual es la siguiente:

$$
\% \text { Eficiencia }=\frac{(\text { Concentración inicial })-(\text { Concentración final })}{(\text { Concentración inicial })} * 100
$$

\section{Resultados y discusión}

\subsection{Caracterización del agua residual doméstica}

En la Tabla 2 se muestran los resultados de la caracterización inicial del afluente, junto con los criterios de límites máximos permisibles para descargas en cuerpos de agua dulce establecidos en el Anexo 1 TULSMA: Norma de calidad ambiental y de descarga de efluentes al recurso agua, Tabla 9: Límites de descarga a un cuerpo de agua dulce.

Tabla 2. Características fisicoquímicas del agua residual doméstica previa a tratamiento

\begin{tabular}{|c|c|c|c|}
\hline Parámetro & Unidad & Valor obtenido & LMP \\
\hline $\mathrm{DBO}_{5}$ & $\mathrm{mg} / \mathrm{l}$ & 74 & 100 \\
\hline $\mathrm{DQO}$ & $\mathrm{mg} / \mathrm{l}$ & 130.59 & 130 \\
\hline $\mathrm{SST}$ & $\mathrm{mg} / \mathrm{l}$ & 1468 & 1600 \\
\hline $\mathrm{ST}$ & $\mathrm{mg} / \mathrm{l}$ & 1987 & $6-9$ \\
\hline $\mathrm{pH}$ & & 6.90 & Condición natural \pm 3 \\
\hline Temperatura & ${ }^{\circ} \mathrm{C}$ & 22.2 & \\
\hline
\end{tabular}

Nota: Reforma del Texto Unificado de Legislación (2015)

Se puede constatar que el agua residual doméstica sin tratar presentó un valor de $\mathrm{DBO}_{5} \mathrm{y}$ DQO igual a 74 y 130.59 mg/l respectivamente, es decir, se encuentra dentro del límite máximo permisible que establece la normativa ambiental vigente del Ecuador. Sin embargo, los sólidos 
suspendidos y sólidos totales se encontraron por encima del límite establecido, con valores de 1468 y 1987 mg/l respectivamente. Además, el pH del agua se encuentra dentro del rango 6-9 al igual que la temperatura con $22.2^{\circ} \mathrm{C}$.

En consecuencia, a pesar de que se encuentra por debajo del límite máximo permisible para $\mathrm{DBO}_{5}$ y DQO, la acumulación diaria de este tipo de agua residual doméstica representa un grave problema, porque la presencia de materia orgánica en exceso y organismos patógenos, así como su constante acumulación, podrían traer como resultado la aparición de enfermedades en los habitantes que residen en la zona de estudio. De acuerdo con Sosa et al. (2014), la ausencia de personal capacitado y los elevados costos de operación y mantenimiento que no permiten un tratamiento a tiempo de estas aguas podrían ocasionar polución ambiental y afectaciones en la salud.

Además, con base en la clasificación propuesta por Metcalf y Eddy (1995), en la cual se detalla la composición típica de un agua residual doméstica, se explica que las características iniciales del afluente sin tratamiento corresponden a un agua residual de concentración baja para $\mathrm{DBO}_{5}$ y DQO, así como de concentración alta en cuanto a los sólidos totales y suspendidos.

\subsection{Adaptación de Eisenia foetida al agua residual doméstica}

En la Figura 4 se muestran los resultados del proceso de adaptación de la lombriz Eisenia foetida durante diez días. Se puede observar que la temperatura en el proceso de adaptación se mantuvo entre 22 y $23^{\circ}$ C. Esto concuerda con lo expuesto por Baumgartner et al. (2013), quienes dicen que la temperatura óptima para el desarrollo de la Eisenia foetida en cualquier sustrato debe oscilar entre 20 y $25^{\circ} \mathrm{C}$. Asimismo, De Lima Rodrigues et al. (2017) indican que este tipo de lombriz puede reproducirse en cualquier parte del mundo mientras sea en un lugar con climas templados con temperaturas promedio superior a los $20^{\circ} \mathrm{C}$.

Figura 4. Adaptación de Eisenia foetida al agua residual doméstica durante 10 días

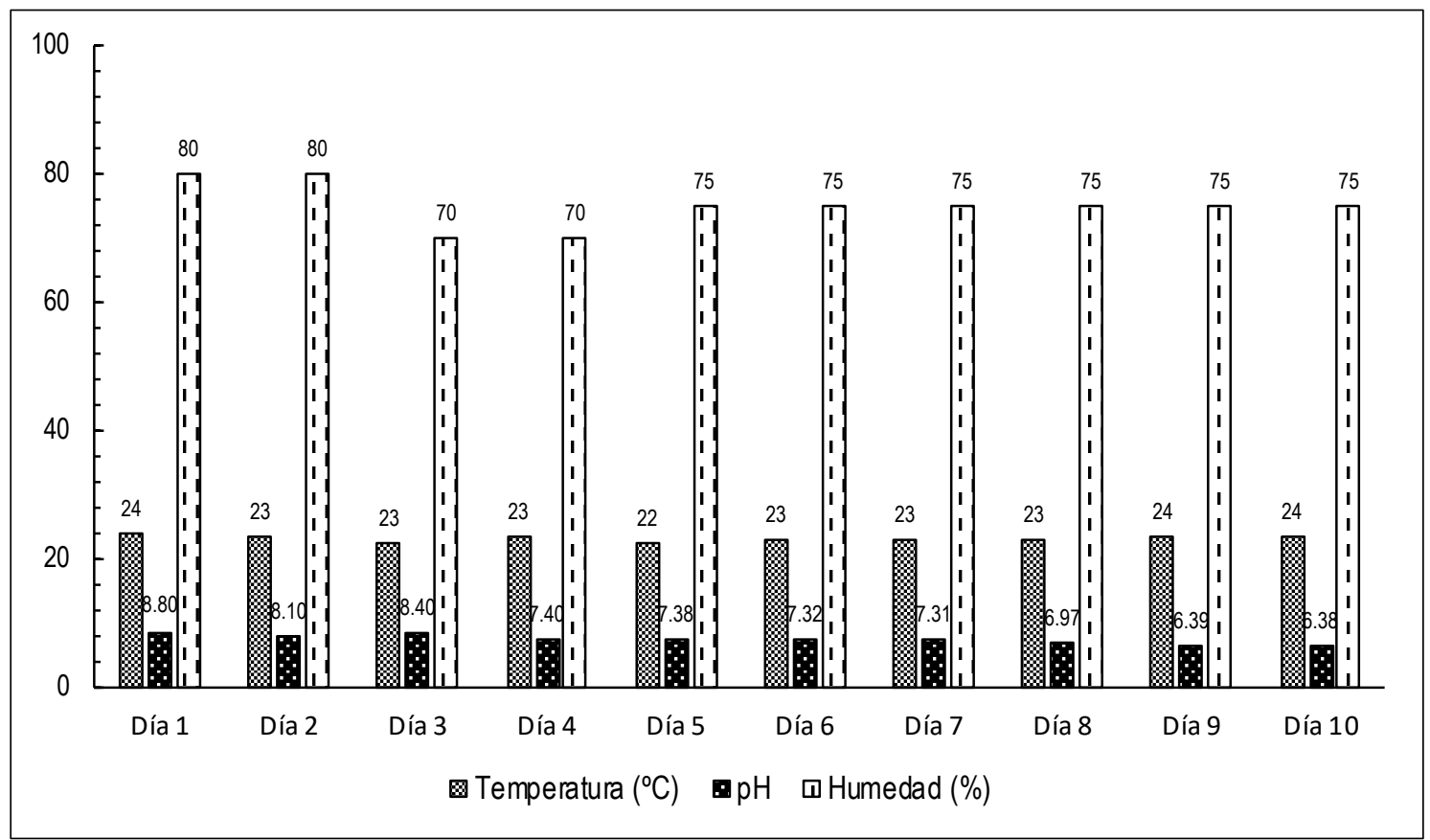


Asimismo, el pH fue superior a 8 los tres primeros días, disminuyendo consecutivamente hasta experimentar un descenso final de 6.38. Pramanik y Chung (2011) dicen que las lombrices Eisenia foetida están adaptadas para sobrevivir en entornos difíciles y en condiciones moderadamente ácidas o alcalinas, con un valor de pH que oscila entre 4.5 y 9 . Sin embargo, de acuerdo con Paico (2017), las condiciones ideales y favorables para su hábitat comprende un $\mathrm{pH}$ entre 6.5-7.5, porque fuera de ese rango la lombriz entra en un estado de latencia, lo cual puede ocasionar la muerte de esta. En adición, se produjeron dos decesos debido al manejo del experimento.

El porcentaje de humedad fue de $80 \%$ los dos primeros días. Posteriormente, el tercer y cuarto día descendió a 70 \% y el resto del periodo de adaptación se mantuvo en un 75 \%. Loro (2018) explica que las condiciones de humedad más favorables para la Eisenia foetida oscilan entre $70 \%-80 \%$, puesto que los ambientes con humedades fuera de este rango pueden ser mortales para las lombrices.

\subsection{Caudal y tiempo de retención hidráulico}

Para medir el caudal, se llevaron a cabo tres repeticiones con tres tipos de apertura de la válvula de control, a partir de la cual se obtuvo un caudal promedio por cada tratamiento para así obtener resultados más exactos del TRH, los cuales se muestran en la Tabla 3.

En la experimentación realizada y con base en los caudales calculados in situ, se obtuvo un TRH de 0.92 h, 0.49 h y $0.15 \mathrm{~h}$ para el primer, segundo y tercer tratamiento aplicado respectivamente (Figura 5). Es por ello que, de acuerdo con las observaciones respectivas, se pudo ver que $T_{3}$ provocó la acumulación del agua en la primera capa (aserrín + Eisenia foetida), lo cual, teniendo en cuenta a Caicedo (2017), no es favorable para tratar el agua residual, debido a que las lombrices solo necesitan un 70 a 80 \% de humedad para una óptima degradación de la materia orgánica.

Tabla 3. Tiempo de retención hidráulico de acuerdo con los caudales obtenidos

\begin{tabular}{|c|c|c|}
\hline Tratamiento & Q (l/s) & TRH (h) \\
\hline$T_{1}(25 \%$ apertura válvula de control $)$ & $1.8 \times 10^{-2}$ & 0.92 \\
\hline$T_{2}(50 \%$ apertura válvula de control $)$ & $3.4 \times 10^{-2}$ & 0.49 \\
\hline$T_{3}(100 \%$ apertura válvula de control $)$ & $1.13 \times 10^{-}$ & 0.15 \\
\hline
\end{tabular}

La aplicación de $T_{2}$ no ocasionó ningún tipo de acumulación de agua. Sin embargo, la eficiencia de remoción no fue la más idónea; mientras que con $T_{1}$, el agua se distribuyó homogéneamente por el lombrifiltro sin producir inundaciones y en un tiempo prudente. Además, fue con este que se obtuvieron los mejores resultados de remoción de la materia orgánica.

En efecto, Manyuchi et al. (2019) argumentan que el tiempo de retención hidráulico influye directamente en el rendimiento del lombrifiltro, pues a mayor tiempo de retención mayor será la eficiencia de remoción. De esta manera, una prolongación de tiempo de retención hidráulico favorece la calidad de biomasa y evita la aglomeración de materia, la cual podría disminuir la eficiencia del tratamiento (Kumar et al., 2016). 
Figura 5. Cálculo del caudal y TRH en el lombrifiltro terminado

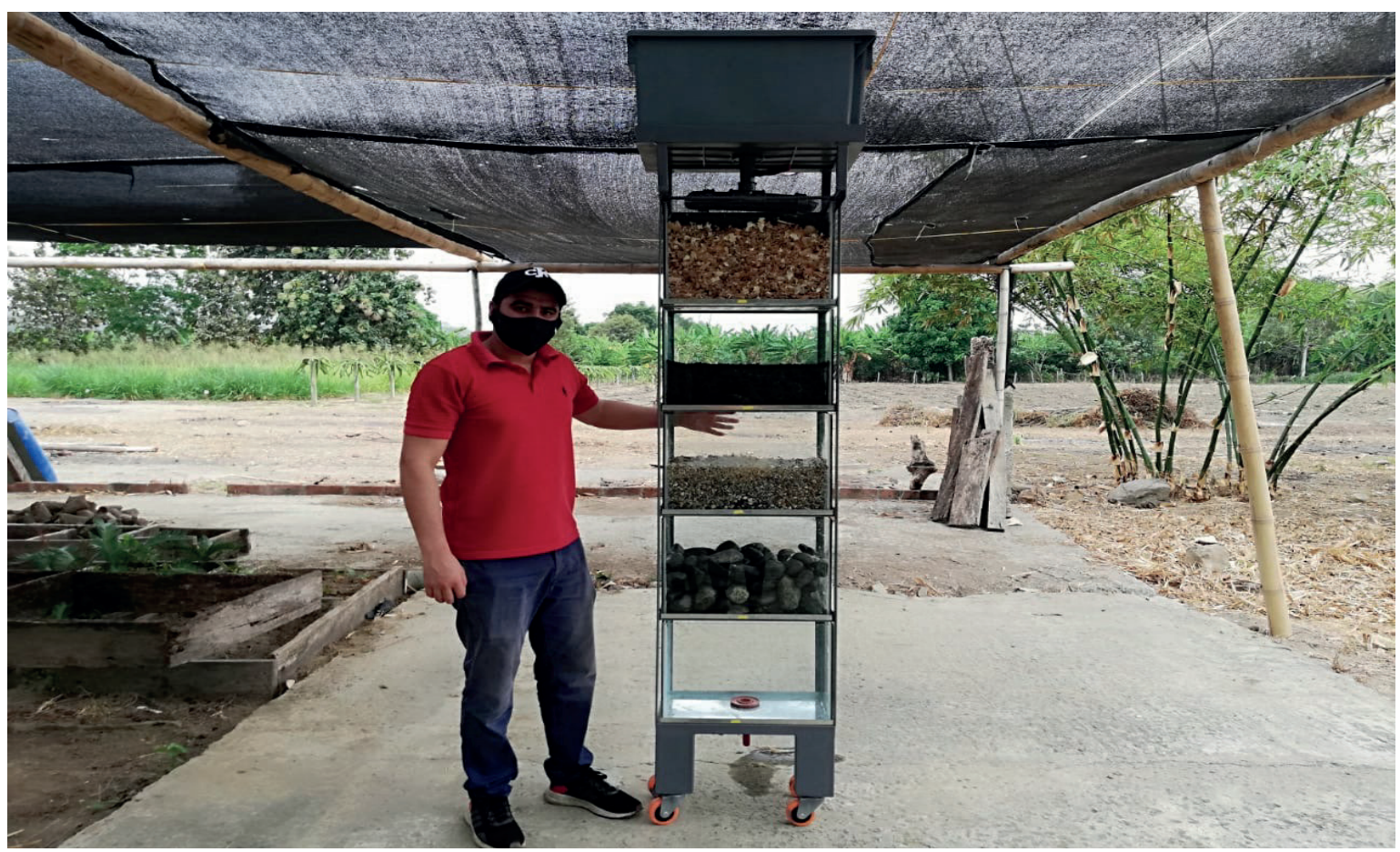

\subsection{Determinación de la eficiencia (porcentaje de remoción)}

\section{A. $\mathrm{DBO}_{5}$ Y DQO}

Se determinó la eficiencia del lombrifiltro mediante los valores de concentración de los diferentes parámetros del agua que ingresó al sistema (afluente) y la del agua que salió después del tratamiento (efluente).

Según se muestra en la Tabla 4, los resultados son corroborados con el análisis de varianza (ANOVA), determinando que existen grandes similitudes en la capacidad de remoción de materia orgánica; es decir, no existen diferencias significativas entre los tres tratamientos aplicados para la remoción de $\mathrm{DBO}_{5}$ y DQ0, ya que el p-valor es de 0.3170 con un nivel de significancia del $95 \%$.

Tabla 4. Análisis de varianza (ANOVA) para demanda bioquímica de oxígeno (DBO5) y demanda química de oxígeno (DQO)

\begin{tabular}{|c|c|c|c|c|c|}
\hline Variable & $\mathbf{N}$ & $\mathbf{R}^{\mathbf{2}}$ & $\mathbf{R}^{\mathbf{2}} \mathbf{A j}$ & $\mathbf{C V}$ & \\
\hline Eficiencia & 9 & 0.32 & 0.09 & 1.95 & \\
\hline \multicolumn{7}{|c|}{ Cuadro de análisis de la varianza (SC tipo III) } \\
\hline F.V. & SC & gl & CM & F & p-valor \\
\hline Modelo & 2.84 & 2 & 1.42 & 1.40 & 0.3170 \\
\hline Tratamiento & 2.84 & 2 & 1.42 & 1.40 & 0.3170 \\
\hline Error & 6.07 & 6 & 1.01 & & \\
\hline Total & 8.91 & 8 & & & \\
\hline
\end{tabular}


Tal como se muestra la Figura 6, y después de obtener como resultado la remoción en porcentajes respecto a los tiempos de retención hidráulicos (TRH), la máxima eficiencia de remoción de $\mathrm{DBO}_{5}$ correspondió a $\mathrm{T}_{1^{\prime}}$ con una media de $52.25 \%$, seguido por $\mathrm{T}_{2}$ y $\mathrm{T}_{3^{\prime}}$ con 51.35 \% y $50.90 \%$ respectivamente. Por esta razón, todos los tratamientos reportaron concentraciones finales inferiores a $40 \mathrm{mg} / \mathrm{l}$.

Las eficiencias de materia orgánica medida como $\mathrm{DBO}_{5}$ son inferiores a las publicadas por Saboya (2018), aplicando también el sistema Tohá con Eisenia foetida, cuyos porcentajes medios fueron de $91 \%$. Sin embargo, esos resultados se asemejan a los obtenidos por Huiza y Ordoñez (2018), los cuales determinaron la eficiencia de un lombrifiltro utilizando la técnica de pared caliente para tratar aguas residuales domésticas, con la que confirman una eficiencia de remoción superior al 50 \% de la carga orgánica inicial.

La Figura 7 se muestra que la DQO obtuvo el mismo porcentaje de remoción en comparación con la $\mathrm{DBO}_{5}$ para los tres tratamientos, con la diferencia de que la concentración en cada uno de ellos fue mayor, con 62.35, 63.53 y $64.12 \mathrm{mg} / \mathrm{l}$ para $T_{1} \mathrm{~T}_{2}$ y $\mathrm{T}_{3}$ respectivamente. Cabe destacar que todas las réplicas obtuvieron concentraciones menores al límite máximo permisible establecido en la normativa ambiental vigente, específicamente para descargas de efluentes en un cuerpo de agua dulce.

En varios estudios relacionados con el tratamiento de aguas residuales, los porcentajes de remoción se aproximan a los obtenidos. Tal es el caso de Mejía et al. (2017), quienes mediante un prototipo a escala de laboratorio lograron obtener una remoción de 53 \% de QDO en aguas residuales domésticas.

Figura 6. Porcentaje de remoción de DBO5

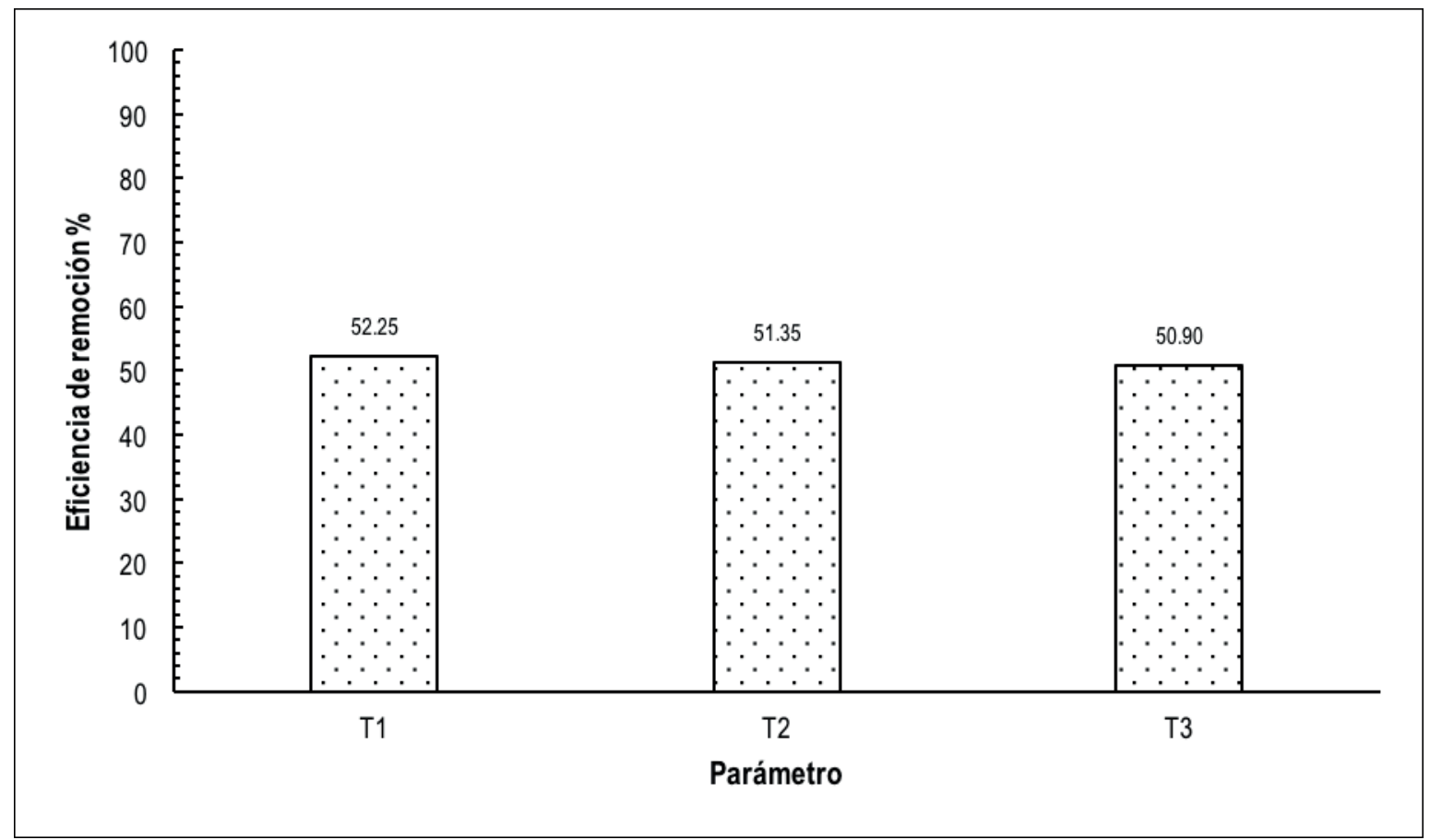


Figura 7. Porcentaje de remoción de DQO

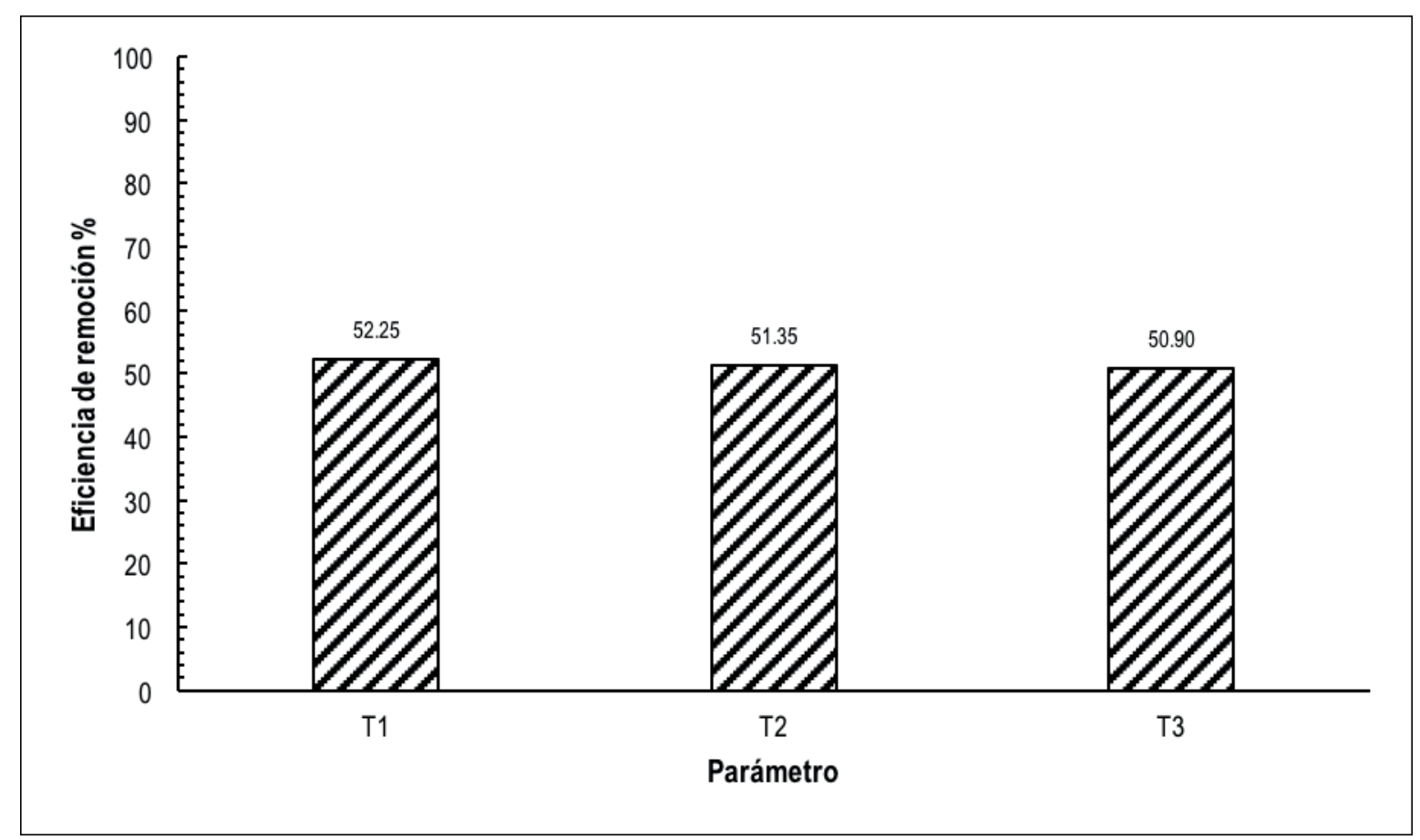

Por su parte, como afirman Asthana et al. (2016), uno de los principales motivos por los que existe una reducción significativa de la DQO es el metabolismo de los macro y microorganismos heterótrofos aerobios y anaerobios que, a través de compuestos orgánicos del agua, producen biomasa, así como a las reacciones químicas de óxido reducción, mediante las cuales sucede dicho proceso.

\section{B. Sólidos SUSPENDIDOS TOTALES (SST)}

Al realizar un análisis de varianza para SST, se comprueba que existen diferencias significativas entre los tres tratamientos aplicados ( $p<0.0001)$. Es decir, las diferencias de porcentajes de remoción no se deben al azar y, por lo tanto, se aprueba la hipótesis alternativa, porque existe un notable efecto de los caudales aplicados en el proceso de tratamiento del agua residual (Tabla 5).

Tabla 5. Análisis de varianza (ANOVA) para sólidos suspendidos totales (SST)

\begin{tabular}{|c|c|c|c|c|c|}
\hline Variable & $\mathbf{N}$ & $\mathbf{R}^{\mathbf{2}}$ & $\mathbf{R}^{\mathbf{2}} \mathbf{A j}$ & $\mathbf{C V}$ & \\
\hline Eficiencia & 9 & 0.99 & 0.99 & 2.56 & \\
\hline \multicolumn{7}{|c|}{ Cuadro de análisis de la varianza (SC tipo III) } \\
\hline F.V. & SC & gl & CM & F & p-valor \\
\hline Modelo & 1634.11 & 2 & 817.06 & 524.03 & $<0.0001$ \\
\hline Tratamiento & 1634.11 & 2 & 817.06 & 524.03 & $<0.0001$ \\
\hline Error & 9.35 & 6 & 1.56 & & \\
\hline Total & 1643.47 & 8 & & & \\
\hline
\end{tabular}


De esta manera, al realizar una comparación de medias mediante la prueba de Tukey, con un $5 \%$ de probabilidad de error, se identificaron tres grupos significativamente diferentes, de los cuales se observa claramente a $T_{1}$ como el más eficiente, con una eficiencia media de $66.74 \%$ de remoción (Tabla 6).

Tabla 6. Prueba de Tukey para sólidos suspendidos totales (SST)

\begin{tabular}{|c|c|c|c|c|c|c|}
\hline Tratamiento & Medias & n & E.E. & \multicolumn{3}{|l|}{} \\
\hline$T_{3}$ & 34.33 & 3 & 0.72 & $A$ & & \\
\hline$T_{2}$ & 45.10 & 3 & 0.72 & & $B$ & \\
\hline$T_{1}$ & 66.74 & 3 & 0.72 & & & $C$ \\
\hline
\end{tabular}

Nota: Test: Tukey Alfa $=0.05$ DMS $=3.12820$

Medias con una letra común no son significativamente diferentes $(p>0.05)$

En consideración con el comportamiento de los tratamientos frente a la eficiencia de remoción de SST, en la Figura 8 se muestra que T, logró obtener los mejores resultados alcanzando una media de $66.74 \%$, con una concentración final de 488.33 mg/l, la cual se asemeja a los reportes publicados por Cáceres et al. (2018), quienes consiguieron una eficiencia del 66.67 \%.

Figura 8. Porcentaje de remoción de SST

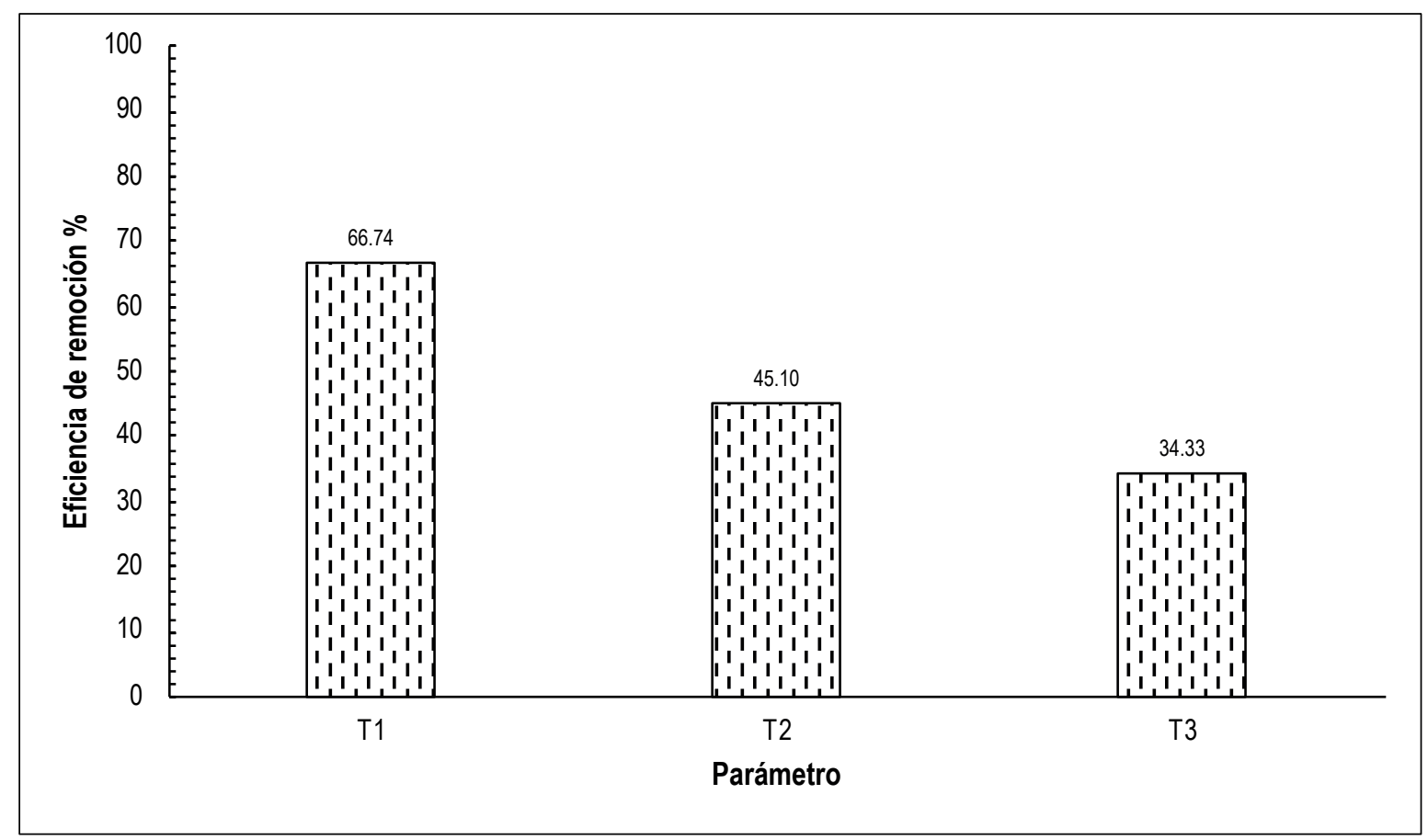

Asimismo, los porcentajes de remoción de este parámetro en los tres tratamientos aplicados son superiores a los reportados por Loro (2018), el cual obtuvo un 40 \% de remoción debido a que la capa de estiércol vacuno y aserrín aumentaron la carga de SST a la salida del lombrifiltro. 


\section{Sólidos totales (ST)}

La Tabla 7 muestra el ANOVA correspondiente al parámetro ST, en la cual se aprecia una diferencia estadísticamente significativa entre los tratamientos, dado que el p-valor es menor a 0.05.

Tabla 7. Análisis de varianza (ANOVA) para sólidos totales (ST)

\begin{tabular}{|c|c|c|c|c|c|}
\hline Variable & $\mathbf{N}$ & $\mathbf{R}^{\mathbf{2}}$ & $\mathbf{R}^{\mathbf{2}} \mathbf{A j}$ & $\mathbf{C V}$ & \\
\hline Eficiencia & 9 & 1.00 & 1.00 & 1.82 & \\
\hline \multicolumn{7}{|c|}{ Cuadro de análisis de la varianza (SC tipo III) } \\
\hline F.V. & SC & gl & CM & F & p-valor \\
\hline Modelo & 1365.03 & 2 & 682.51 & 1528.36 & $<0.0001$ \\
\hline Tratamiento & 1365.03 & 2 & 682.51 & 1528.36 & $<0.0001$ \\
\hline Error & 2.68 & 6 & 0.45 & & \\
\hline Total & 1367.71 & 8 & & & \\
\end{tabular}

En la Tabla 8 se detalla la prueba de Tukey al 5 \% de probabilidad de error, constatando nuevamente que existe diferencia significativa entre la media de porcentajes de remoción entre un tratamiento y otro. Por tanto, se comprueba que los caudales aplicados, así como los TRH tienen un aporte estadísticamente significativo en la remoción de ST.

Tabla 8. Prueba de Tukey para sólidos totales (ST)

\begin{tabular}{|c|c|c|c|c|c|c|}
\hline Tratamiento & Medias & n & E.E. & \multicolumn{2}{|l|}{} \\
\hline$T_{3}$ & 23.12 & 3 & 0.39 & A & & \\
\hline$T_{2}$ & 33.94 & 3 & 0.39 & & $B$ & \\
\hline$T_{1}$ & 52.91 & 3 & 0.39 & & & C \\
\hline
\end{tabular}

Nota: Test: Tukey Alfa=0.05 DMS=1.67414

Medias con una letra común no son significativamente diferentes ( $p>0.05$ )

De acuerdo con la Figura 9, T, obtuvo la mayor eficiencia de remoción de sólidos totales, obteniendo una media de $52.91 \%$ con una concentración final equivalente a $935.67 \mathrm{mg} / \mathrm{l}$. Asimismo, se observó un promedio de 33.94 y 23.12 \% de eficiencia de remoción para $T_{2}$ y $T_{3^{\prime}}$ con concentraciones finales que superan el límite máximo permisible establecido en la normativa ambiental vigente, las cuales fueron de 1312.67 y $1527.67 \mathrm{mg} / \mathrm{l}$ respectivamente.

Teniendo en cuenta a Xing et al. (2015), la disminución significativa de sólidos totales se atribuye a la actividad de las lombrices de tierra en la bioconversión del material sólido del agua residual en vermicompost, lo cual coincide con Singh et al. (2017), quienes confirman que los sólidos retenidos en el lecho filtrante de aserrín son consumidos por las lombrices de tierra y convertidos en humus y, por lo tanto, no hay generación de lodo como se ve en otras tecnologías de tratamiento de aguas residuales. Por tal motivo, los sólidos de aguas residuales compostados por la Eisenia foetida pueden ser útiles aditivos para el suelo ya que ayudan a mejorar su fertilidad (Sinha et al., 2008). 
Figura 9. Porcentaje de remoción de ST

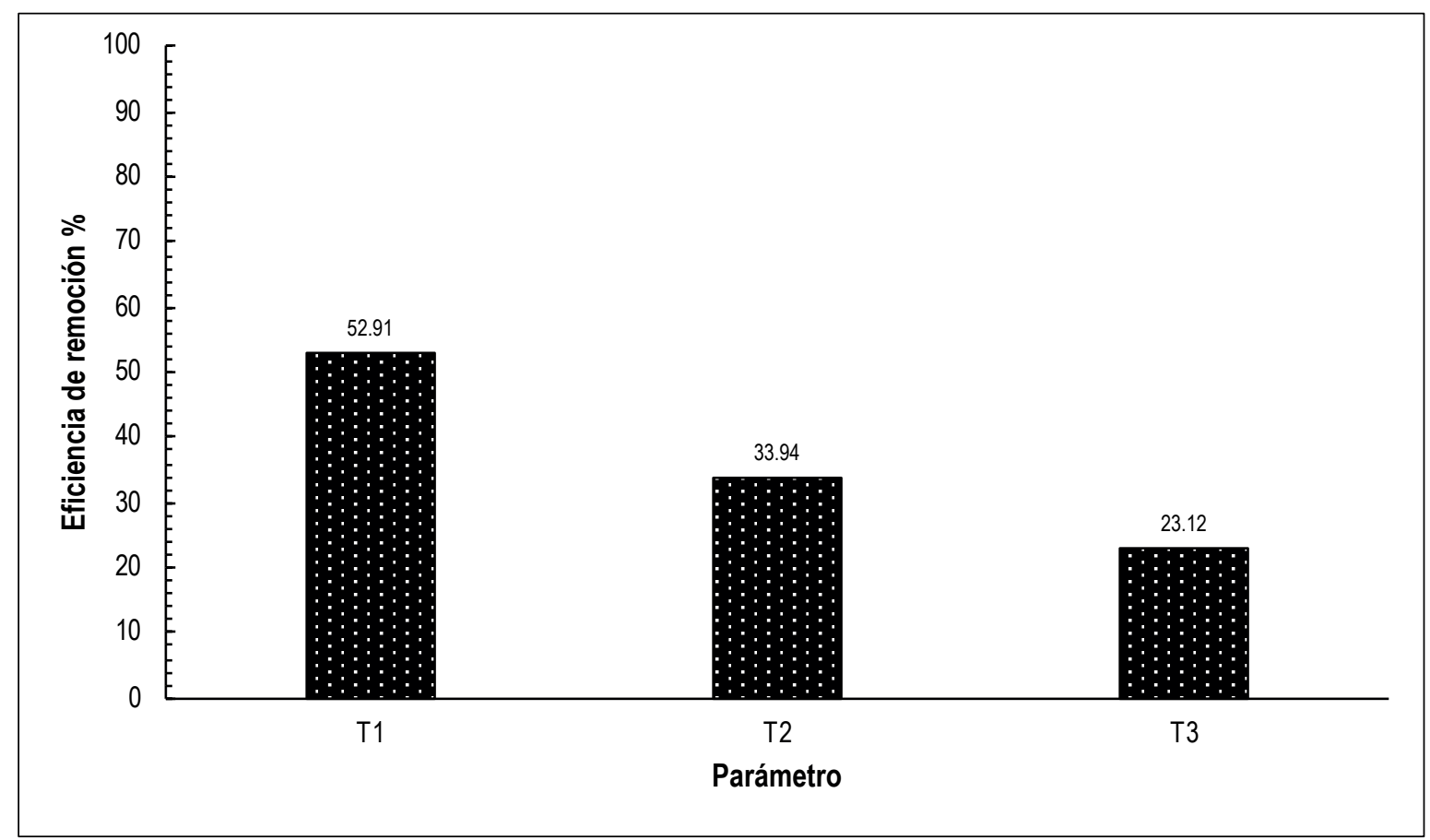

En concordancia con los análisis descritos, se comprueba que $T_{1}$ con un caudal de 1.8×10${ }^{2} \mathrm{l} / \mathrm{s}$ y un TRH de $0.92 \mathrm{~h}$ obtuvo la mayor eficiencia de remoción para $\mathrm{DBO}_{5^{\prime}}, \mathrm{DQO}$, SST y ST, concluyendo que mientras más tiempo tarda el agua residual doméstica en descender por los lechos filtrantes, más eficiente es la remoción de materia orgánica de esta

\section{D. $\mathrm{PH}$}

El seguimiento del comportamiento del pH es un factor de suma importancia en los procesos de transformación química y biológica para tratamientos de aguas residuales (James et al., 2020). En la Figura 10 se presenta el seguimiento de este parámetro observando que en el agua residual sin tratamiento con el lombrifiltro fue de 6.9; mientras que el agua tratada no presentó variaciones bruscas con un pH entre 6.32 y 7.32 para los diferentes tratamientos. Es decir, el valor del pH del agua residual tratada fue prácticamente neutralizado por las lombrices Eisenia foetida en el lombrifiltro, manteniendo un promedio de 6.8.

Similar comportamiento reportó el estudio de Adugna et al. (2019) en el proceso de vermifiltración, en el cual, durante el tratamiento biológico, el pH se estabilizó con rangos similares.

En el estudio de Ghasemi et al. (2019), los valores de pH observados en la entrada y la salida con una tasa de carga de $2 \mathrm{~m}^{3}$ día$^{-1}$ resultaron ser en el rango de $6.75 \pm 0.15$ y $7.42 \pm 0.15$ con un valor medio de pH de $7.07 \pm 0.15$ y $6.94 \pm 0.2$ y $7.78 \pm 0.2$, con un valor medio de $7.78 \pm 0.2$, respectivamente. Durante el proceso de tratamiento, el pH aumentó inicialmente y luego se asentó en el rango neutro, lo que confirmó notablemente la capacidad inherente de las lombrices de aumentar el nivel de mineralización del compuesto orgánico a $\mathrm{CO}_{2}$ y otras sales mineralizadas. Asimismo, estos autores indican que la ligera diferencia entre el valor de $\mathrm{pH}$ de entrada y de salida está relacionada con la eliminación de biocontaminantes por parte de las lombrices y su conversión posterior en vermicompost. 
Figura 10. Comportamiento del pH del agua residual doméstica tratada

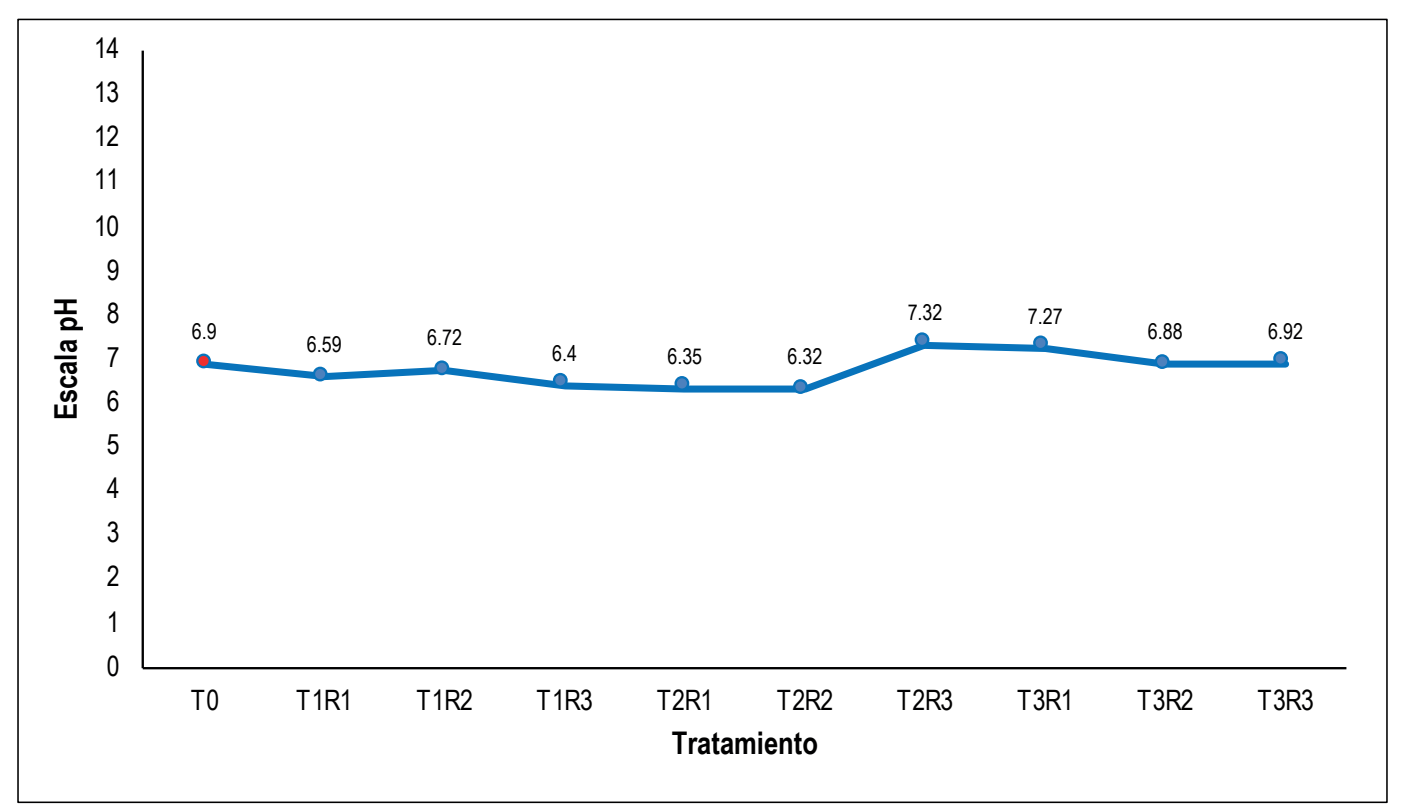

\section{e. Temperatura}

En el tratamiento de aguas residuales por vermifiltración, la eficiencia de remoción de materia orgánica depende, en gran medida, del metabolismo microbiano (Baumgartner, 2013). De acuerdo con la Figura 11, se puede ver que la temperatura se mantuvo entre 22.1 y $22.7^{\circ} \mathrm{C}$. En la investigación de Ramón et al. (2015), así como en la de Coronel (2015), no se consideraron claramente los efectos del tratamiento con la lombriz Eisenia foetida sobre este parámetro.

Figura 11. Comportamiento de la temperatura del agua residual doméstica tratada

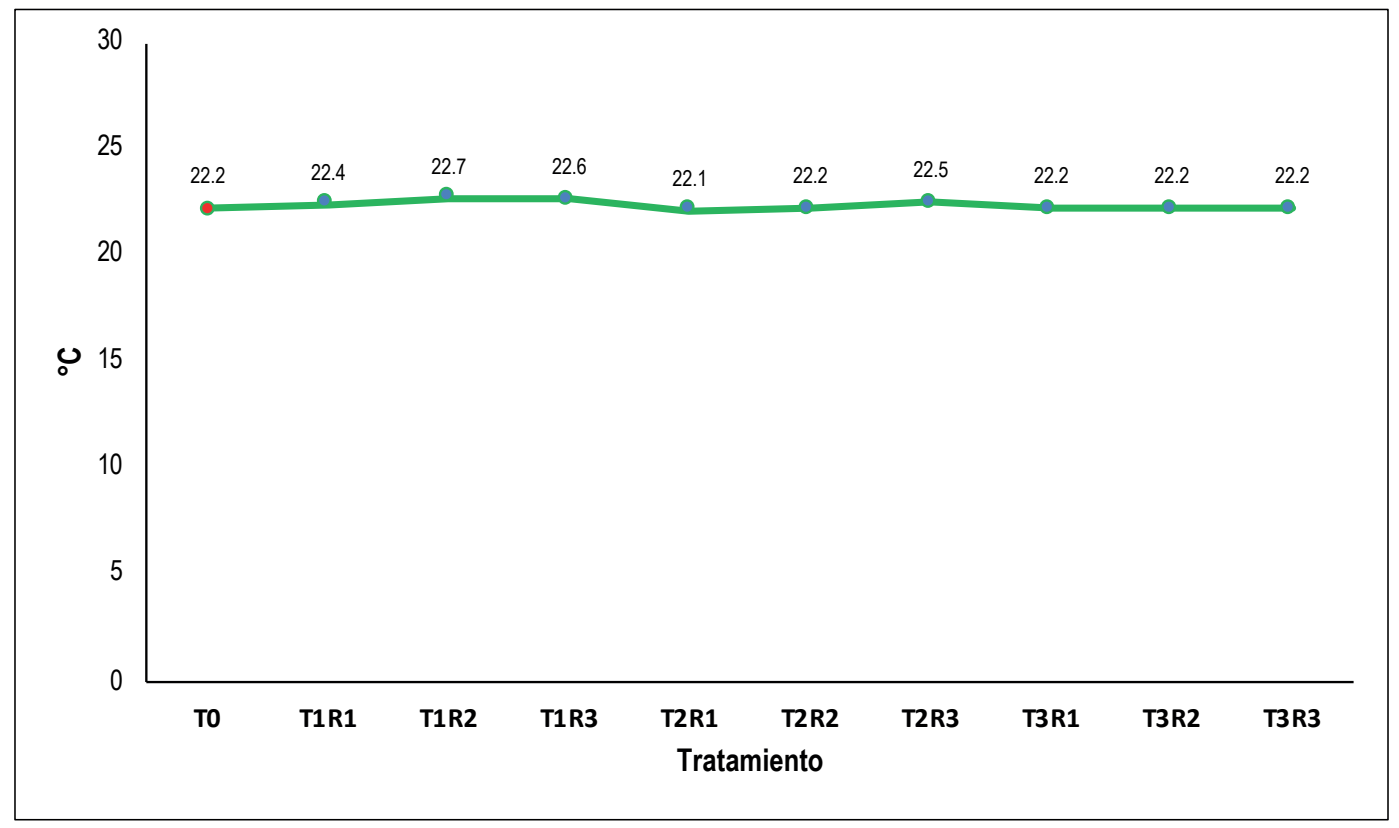


Loro (2018) afirma que la temperatura es un parámetro de vital importancia, el cual debe ser monitoreado constantemente, ya que determina las condiciones esenciales para el desarrollo de microorganismos y de la Eisenia foetida en las reacciones químicas, pues su actividad metabólica se acelera con la temperatura y viceversa. Asimismo, de acuerdo con Jin et al. (2016), las especies de la lombriz de tierra Eisenia foetida favorecen las partículas orgánicas, toleran la fluctuación de la temperatura y se adaptan al ambiente húmedo. En comparación con otras especies, estas poseen una mayor tolerancia a la fluctuación de la temperatura y a la alta humedad, así como una mayor reproducción.

\section{Conclusiones y recomendaciones}

Se obtuvo un caudal de agua residual doméstica con un promedio de $5.18 \mathrm{~m}^{3} /$ día, la cual, a través de los análisis de laboratorio, tenía una elevada concentración de materia orgánica en cuanto a SST y ST. Sin embargo, los parámetros de $\mathrm{DBO}_{5}$ y DQO se encontraron por debajo del límite máximo permisible establecido en la legislación ambiental ecuatoriana.

Los diferentes caudales aplicados en el lombrifiltro influyeron significativamente en la eficiencia de remoción de materia orgánica, existiendo una variabilidad en el porcentaje de remoción de SST y ST. Mientras que la $\mathrm{DBO}_{5}$ y DQO no presentaron diferencias estadísticamente significativas entre un tratamiento y otro ya que el p-valor fue mayor a 0.05 .

El $\mathrm{T}_{1}$, con un caudal de $1.8 \times 10^{-2} \mathrm{l} / \mathrm{s}$ y un TRH de $0.92 \mathrm{~h}$, obtuvo los mejores resultados de eficiencia, con medias de $52.25 \%$ para DBO D DQO $_{5} 66.74$ y $52.91 \%$ para SST y ST respectivamente, y un $\mathrm{pH}$ y temperatura promedio de 6.8 y $22.3^{\circ} \mathrm{C}$. Por esta razón, se identificó que mientras mayor es el TRH, la eficiencia de remoción de materia orgánica que tiene el lombrifiltro aumenta.

La implementación de lombrifiltros a base de Eisenia foetida para remover materia orgánica en aguas residuales domésticas representa un sistema ideal para las zonas rurales, debido a sus características de eficiencia, rentabilidad y sostenibilidad ecológica.

\section{Referencias}

Reforma del Texto Unificado de Legislación Secundaria (TULSMA), Acuerdo Ministerial 097-A, R. O. No. 387. Anexo 1. Tabla 9. (2015).

Adugna, A., Andrianisa, H., Konate, Y., \& Maiga, A. (2019). Fate of Filter Materials and Microbial Communities During Vermifiltration Process. Journal of Environmental Management, 242, 98-105. https://doi.org/10.1016/j.jenvman.2019.04.076

Agua. Calidad del agua. Muestreo. Manejo y conservación de muestras, NTE INEN 2169:2013. (2013a). http://www.trabajo.gob.ec

Agua. Calidad del agua. Muestreo. Técnicas de muestreo, NTE INEN 2176:2013. (2013b). http://www.trabajo.gob.ec

Alcívar, U., Dueñas, A., Sacon, E., Bravo, L., \& Villanueva, G. (2016). Influencia de los tipos de secado para la obtención de harina de lombriz roja californiana (Eisenia foetida) a escala piloto. Tecnología Química, 36(2),.187-196. https://www.redalyc.org/jatsRepo/4455/445546335007/index.html

Asthana, M., Kumar, A., \& Sharma, B. S. (2016). Wastewater Treatment: Principles and Applications of Environmental Biotechnology for a Sustainable Future, 173-232. https://doi.org/10.1007/978-98110-1866-4_6

Baumgartner, R. (2013). Developing a Testing Protocol for Vermifiltration-based Onsite Wastewater Treatment Systems (VOWTS), 1-60. http://researchrepository.murdoch.edu.au/id/eprint/16946 
Cáceres, D., Calisaya, G., \& Bedoya, E. (2018). Eficiencia de la lombriz roja californiana (Eisenia foetida) en el tratamiento de aguas residuales domésticas. Revista Ciencia y Tecnología para el Desarrollo, 4, 13-23. https://revistas.ujcm.edu.pe/index.php/rctd/article/view/115

Caicedo, J. (2017). Diseño, construcción y evaluación de un prototipo biológico compuesto de Eisenia foetida y agave filifera, para el tratamiento de aguas residuales en la granja del Ministerio de Agricultura, Ganadería, Acuacultura y Pesca, Riobamba 2015 [Tesis de pregrado, Escuela Superior Politécnica de Chimborazo]. Repositorio Institucional ESPOCH. http://dspace.espoch.edu. ec/handle/123456789/6321

Coronel, N. (2015). Diseño e implementación a escala de un biofiltro Tohá en la ESPOCH para la depuración de aguas residuales domésticas procedentes de la comunidad Langos La Nube [Tesis de pregrado, Escuela Superior Politécnica de Chimborazo]. Repositorio Institucional ESPOCH. http:// dspace.espoch.edu.ec/handle/123456789/4802

De Anda, J. (2017). Saneamiento descentralizado y reutilización sustentable de las aguas residuales municipales en México. Sociedad y Ambiente, 14(1), 119-143. http://www.scielo.org.mx/pdf/sya/ n14/2007-6576-sya-14-119.pdf

De Lima Rodrigues, A., Mesak, C., Silva, M., Silva, G., Leandro, W., \& Malafaia, G. (2017). Organic Waste Vermicomposting through The Addition of Rock Dust Inoculated with Domestic Sewage Wastewater. Journal of Environmental Management, 196, 651-658. https://doi.org/10.1016/j.jenvman.2017.03.072

Dharwal, M., Parashar, D., Shuaibu, M. S., Abdullahi, S. G., Abubakar, S., \& Bala, B. B. (2020). Water pollution: Effects on Health and Environment of Dala LGA, Nigeria. Materials Today: Proceedings, S/p. Corpus ID: 229397024. https://doi.org/10.1016/j.matpr.2020.10.496

García, Z. (2012). Comparación y evaluación de tres plantas acuáticas para determinar la eficiencia de remoción de nutrientes en el tratamiento de aguas residuales domésticas [Tesis de pregrado, Universidad Nacional de Ingeniería]. Repositorio UNI-Tesis. http://cybertesis. uni. edu. pe/handle/uni/1292.

Ghasemi, S., Mirzaie, M., Hasan-Zadeh, A., Ashrafnejad, M., Hashemian, S. J., \& Shahnemati, S. R. (2019). Design, Operation, Performance Evaluation and Mathematical Optimization of a Vermifiltration Pilot Plan for Domestic Wastewater Treatment. Journal of Environmental Chemical Engineering, 8(1), 103-587. https://doi.org/10.1016/j.jece.2019.103587

Guzmán, T. M., Pérez Chamorro, O., \& Valdés Pérez, O. (2020). Biodegradación de residuos sólidos urbanos utilizando cultivos microbianos y biofiltro estático de Eisenia foetida. Tecnología Química, 40(1), 81-92.

Huiza, J., \& Ordoñez, N. (2018). Eficiencia de lombrifiltro implementando la técnica de pared caliente en el tratamiento de aguas residuales domésticas del centro poblado de Huaylacucho del distrito de Huancavelica. [Tesis de pregrado, Universidad Nacional de Huancavelica]. Repositorio Institucional UNH. http://repositorio.unh.edu.pe/handle/UNH/2429

Chippymol, J., Meenal, S. H., Elakkiya, S., \& Logarshani, S. (2020). Sustainable Environment Through Treatment of Domestic Sewage Using MFC. Materials Today: Proceedings, 37(2), 1495-1502. https://doi.org/10.1016/j.matpr.2020.07.110

Jin, Q., Li, W., \& Li, X. (2016). Effect of Earthworm Eisenia foetida in Constructed Wetland on Purification of Country Wastewater. Procedia Engineering, 154, 406-411. https://doi.org/10.1016/j. proeng.2016.07.505

Kumar, T., Rajpal, A., Arora, S., Bhargava, R., Prasad, K., \& Kazmi, A. (2016). A Comparative Study on Vermifiltration Using Epigeic Earthworm Eisenia fetida and Eudrilus eugeniae. Desalination and Water Treat 57(14), p 6347-6354. https://doi.org/10.1080/19443994.2015.1010230

Lohani, S. P., Khanal, S. N., \& Bakke, R. (2020). A Simple Anaerobic and Filtration Combined System for Domestic Wastewater Treatment. Water-Energy Nexus, 3(1), 41-45. https://doi.org/10.1016/j. wen.2020.03.004 
Loro, A. (2018). Evaluación de la eficiencia del tratamiento secundario de aguas residuales domésticas utilizando un biofiltro con Eisenia foetida y un biofiltro convencional [Tesis de pregrado, Universidad Científica del Sur]. Repositorio UCSUR-Institucional. https://hdl.handle.net/20.500.12805/567

Manyuchi, M. M., Mupoperi, N., Mbohwa, C., \& Muzenda, E. (2019). Treatment of Wastewater Using Vermifiltration Technology. Water Conservation, Recycling and Reuse: Issues and Challenges, 215230. https://doi.org/10.1007/978-981-13-3179-4_12

Mejía, A., Cabrera, M., \& Carrillo, Y. (2017). Remoción de contaminantes orgánicos presentes en agua residual doméstica mediante prototipo a escala de laboratorio. La Granja: Revista de Ciencias de la Vida, 26(2), 72-83. https://doi.org/10.17163/lgr.n26.2017.06

Metcalf \& Eddy. (1995). Ingeniería de aguas residuales: Tratamiento, vertido y reutilización (3. ${ }^{a}$ ed). McGraw Hill.

NBS (National Bureau of Statistics). (2017). Report on the State of Agriculture of China. http://www.stats. gov.cn/english/

Paico, D. (2017). Sistema Tohá, para el tratamiento de aguas residuales de la Universidad Cesar Vallejo. [Tesis de pregrado, Universidad Cesar Vallejo]. Repositorio Institucional UCV. https://hdl.handle.net/20.500.12692/10890

Pramanik, P., \& Chung, Y. (2011). Changes in Fungal Population of Fly Ash and Vinasse Mixture During Vermicomposting by Eudrilus eugeniae and Eisenia fetida: Documentation of Cellulase Isozymes in Vermicompost. Waste Manag, 1(31), 1169-1175. https://doi.org/10.1016/j.wasman.2010.12.017

Priya, A. K., Pachaiappan, R., Kumar, P. S., Jalil, A. A., Vo, D. V. N., \& Rajendran, S. (2021). The War Using Microbes: A Sustainable Approach for Wastewater Management. Environmental Pollution, 275, 116598. https://doi.org/10.1016/j.envpol.2021.116598

Ramón, J., León, J., \& Castillo, N. (2015). Diseño de un sistema alternativo para el tratamiento de aguas residuales urbanas por medio de la técnica de lombrifiltros utilizando la especie Eisenia foetida. Revista Mutis, 5(1), 46-54. https://revistas.utadeo.edu.co/index.php/mutis/article/view/1018/1053

Reyes, J. (2016). Determinación de la eficiencia del aserrín y la fibra de coco utilizados como empaques para la remoción de contaminantes en biofiltros para el tratamiento de aguas residuales. Enfoque UTE, 7(3), 41-56. https://doi.org/10.29019/enfoqueute.v7n3.104

Saboya, X. (2018). Eficiencia del método de lombrifiltro en la remoción de los contaminantes de las aguas residuales domésticas en el Distrito de Chachapoyas-Amazonas. [Tesis de pregrado, Universidad Peruana Unión]. Repositorio UPU. http://repositorio.upeu.edu.pe/handle/UPEU/1123

Salazar, M. (2005). Sistema Tohá: Una alternativa ecológica para el tratamiento de aguas residuales en sectores rurales [Tesis de pregrado, Universidad Austral de Chile]. Repositorio Institucional UACh. http://cybertesis.uach.cl/tesis/uach/2005/bmfcis161s/doc/bmfcis161s.pdf

Samal, K., Dash, R. R., \& Bhunia, P. (2018). A Comparative Study of Macrophytes Influence on Performance of Hybrid Vermifilter for Dairy Wastewater Treatment. Journal of Environmental Chemical Engineering, 6(4), 4714-4726. https://doi.org/10.1016/j.jece.2018.07.018

Singh, R., Bhunia, P., \& Dash, R. (2017). A Mechanistic Review on Vermifiltration of Wastewater: Design, Operation and Performance. Journal of Environmental Management, 1(1), 656-672. https://doi. org/10.1016/j.jenvman.2017.04.042

Singh, R., Bhunia, P., \& Dash, R. R. (2017). A Mechanistic Review on Vermifiltration of Wastewater: Design, Operation and Performance. Journal of Environmental Management, 197, 656-672. https://doi. org/10.1016/j.jenvman.2017.04.042

Sinha, R., Bharambe, G., \& Chaudhari, U. (2008). Sewage Treatment by Vermifiltration with The Synchronous Treatment of Sludge by Earthworms: A Low-Cost Sustainable Technology Over Conventional Systems with Potential for Decentralization. Environmentalist, 28(4), 409-420. https://doi. org/10.1007/s10669-008-9162-8

Sosa, D., Viguera, J., \& Holguín, E. (2014). La biofiltración: Una alternativa sustentable para el tratamiento de aguas residuales. Vidsupra Visión Científica, 6(2), 56-60. https://www.ciidirdurango.ipn.mx 
Sudipti, A., \& Sakshi, S. (2021). Vermifiltration as A Natural, Sustainable and Green Technology for Environmental Remediation: A New Paradigm for Wastewater Treatment Process. Current Research in Green and Sustainable Chemistry, 4(44), 7513-7520. https://doi.org/10.5897/AJB10.811

Tejada, A., Chura, E., \& Apaza, H. (2018). Mejoramiento del sistema de tratamiento de aguas residuales domésticas, modelo Rotoplas para familias del sector rural. Revista Científica de Investigaciones Ambientales, 1(1), 43-54. http://revistas.upsc.edu.pe/journal/index.php/RIAM/article/view/12

Xing, M., Li, X., \& Yang, J. (2015). Treatment Performance of Small-Scale Vermifilter for Domestic Wastewater and Its Relationship to Earthworm Growth, Reproduction and Enzymatic Activity. African Journal of Biotechnology, 9(44), 7513-7520. https://doi.org/10.5897/AJB10.811

Yang, S., Zheng, Y., Mao, Y., Xu, L., Jin, Z., Zhao, M., Kong, H; Huang, X., \& Zheng, X. (2020). Domestic Wastewater Treatment for Single Household Via Novel Subsurface Wastewater Infiltration Systems (SWISs) with NiiMi Process: Performance and Microbial Community. Journal of Cleaner Production, 279, 123434. https://doi.org/10.1016/j.jclepro.2020.123434 